\title{
Promising Diagnostic Accuracy of Plasma GFAP and NfL Within The AD Continuum
}

\section{Tandis Parvizi}

Medical University of Vienna Department of Neurology: Medizinische Universitat Wien Universitatsklinik fur Neurologie https://orcid.org/0000-0001-6989-7539

\section{Theresa Koenig}

Medical University of Vienna Department of Neurology: Medizinische Universitat Wien Universitatsklinik fur Neurologie

\section{Raphael Wurm}

Medical University of Vienna Department of Neurology: Medizinische Universitat Wien Universitatsklinik fur Neurologie

\section{Sara Silvaieh}

Medical University of Vienna Department of Neurology: Medizinische Universitat Wien Universitatsklinik fur Neurologie

\section{Patrick Altmann}

Medical University of Vienna Department of Neurology: Medizinische Universitat Wien Universitatsklinik fur Neurologie

\section{Sigrid Klotz}

Division of Neuropathology and Neurochemistry, Department of Neurology, Medical University of Vienna

\section{Paulus Rommer}

Medical University of Vienna Department of Neurology: Medizinische Universitat Wien Universitatsklinik fur Neurologie

\section{Julia Furtner}

Division of Neuroradiology and Musculoskeletal Radiology, Department of Biomedical Imaging and Image-guided Therapy, Medical University of Vienna

\section{Guenther Regelsberger}

Division of Neuropathology and Neurochemistry, Department of Neurology, Medical University of Vienna Johann Lehrner

Medical University of Vienna Department of Neurology: Medizinische Universitat Wien Universitatsklinik fur Neurologie

\section{Tatjana Traub-Weidinger}

Division of Nuclear Medicine, Department of Biomedical Imaging and Image-guided Therapy, Medical University of Vienna

\section{Ellen Gelpi-Mantius}

Division of Neuropathology and Neurochemistry, Department of Neurology, Medical University of Vienna 
Elisabeth Stögmann ( $\square$ elisabeth.stoegmann@meduniwien.ac.at )

Medical University of Vienna Department of Neurology: Medizinische Universitat Wien Universitatsklinik fur Neurologie https://orcid.org/0000-0003-4688-8482

\section{Research}

Keywords: Alzheimer's disease, biomarker, GFAP, NfL

Posted Date: July 7th, 2021

DOl: https://doi.org/10.21203/rs.3.rs-634853/v1

License: (c) (1) This work is licensed under a Creative Commons Attribution 4.0 International License. Read Full License 


\section{Abstract}

Background: Blood-based biomarkers may add a great benefit in detecting the earliest neuropathological changes in patients with Alzheimer's disease (AD). We examined the utility of neurofilament light chain (NfL) and glial fibrillary acidic protein (GFAP) in plasma and cerebrospinal fluid (CSF) regarding clinical diagnosis and amyloid positivity in an outpatient memory clinic - based cohort.

Methods: In this retrospective analysis, we included a total of 185 patients, 141 patients along clinical the $A D$ continuum, i.e. subjective cognitive decline ( $S C D, n=18)$, mild cognitive impairment $(M C l, n=63), A D$ $(n=60)$ and 44 age-matched healthy controls $(H C)$. CSF and plasma concentrations of NfL and GFAP were measured with single molecule array (SIMOAâ) technology using the Neurology 2-Plex B kit from Quanterix. Amyloid-PET was performed in 75 patients and graded as amyloid positive and negative by visual rating. To assess the discriminatory potential of different biomarkers, age- and sex-adjusted receiver operating characteristic (ROC) curves were calculated and the area under the curve (AUC) of each model was compared using DeLong's test for correlated AUC curves.

Results: We constructed a panel combining plasma NfL and GFAP with known AD risk factors (age+sex+APOE4+GFAP+NfL panel). Using this panel, AUC was $91.6 \%$ for $\mathrm{HC}$ vs. AD, $81.7 \%$ for $\mathrm{HC}$ vs. $\mathrm{MCl}, 85 \%$ for $\mathrm{SCD}$ vs. $\mathrm{AD}, 81.3 \%$ for $\mathrm{SCD}$ vs. $\mathrm{MCl}, 77.7 \%$ for $\mathrm{HC}$ vs. SCD and $72.3 \%$ for $\mathrm{MCl}$ vs. $A D$. In terms of predicting amyloid PET status, we computed an AUC of $88.4 \%$.

Conclusion: The combination of plasma GFAP and NfL with well-established risk factors could contribute crucially to the identification of patients at risk, and thereby facilitate inclusion of patients in clinical trials for disease modifying therapies.

\section{Background}

Alzheimer's disease (AD) represents a frequent neurodegenerative disorder, which leads to a progressive decline in cognitive functions ${ }^{1,2}$. In the last decades, emerging evidence indicates the beginning of neuropathological changes with cerebral accumulation of amyloid-beta $(\mathrm{Ab})$ and neurofibrillary tangles (NFT) 10 to 20 years before manifestation of obvious clinical signs ${ }^{3-5}$. Based on this knowledge, the definition of $A D$ shifted towards a rather biological construct with a better understanding of $A D$ as a disease continuum ${ }^{4,5}$. The preclinical phase of $A D$ defines a stage where early neuropathological changes are present but cognitive deficits are not yet detectable. Regarding the inclusion in clinical trials and the development of disease modifying therapies, it is crucial to identify patients at-risk as early as possible in the evolution of the neuropathological cascade. Therefore, the diagnosis of early phases of $A D$, such as mild cognitive impairment $(\mathrm{MCl})$ due to $A D$ and even stages of subjective cognitive decline $(S C D)$ as a possible late stage of preclinical $A D$, is of particular interest.

Neurodegeneration with early synaptic and consecutive neuronal loss represents an important neuropathological component in the pathophysiology of $A D$ and thus correlates even better with the main symptoms of cognitive decline than do amyloid and tau pathology, although both proteins contribute to 
the neurodegenerative process ${ }^{6,7}$. Considering that positron emission tomography (PET) imaging is a cost-intensive diagnostic biomarker and lumbar puncture is an invasive examination, recent studies have been looking for the possibility to identify reliable fluid biomarkers by conventional blood analysis. The establishment of new and sensitive analytical methods may facilitate this approach. In comparison to already established enzyme-linked immunosorbent assay (ELISA), the usage of ultrasensitive single molecule array (SIMOA $\rightarrow$ ) has proven a superior value for several molecules ${ }^{8}$.

Neurofilament light chain (NfL), a subunit of specific cytoskeletal proteins of neurons, represents a highly proposed biomarker regarding detection of neuronal loss. $\mathrm{NfL}$ is released through axonal damage into the cerebrospinal fluid (CSF) and eventually into the blood ${ }^{9}$. Accumulating data have shown that plasma NfL could be used as a non-invasive biomarker for neurodegeneration, which correlates well with cognitive decline and brain atrophy ${ }^{10-12}$. Studies have found higher plasma $\mathrm{NfL}$ levels in patients with $\mathrm{MCl}$ and $\mathrm{AD}$ in comparison to cognitively unimpaired controls ${ }^{13}$, and estimated a beginning increase in plasma NfL levels about 10 years before AD diagnosis compared to those who remain dementia-free ${ }^{14}$. Another promising biomarker for tracking neurodegenerative changes could be glial fibrillary acidic protein (GFAP), an intermediate filament protein of astrocytes. Neuropathological data have shown a close spatial relationship between reactive astrocytes and amyloid plaques in brain tissue of patients with $A D^{15,16}$. GFAP is known to be involved in the neuroinflammatory cascade of $A D$ pathophysiology. High GFAP concentrations have been detected in the CSF of patients with dementia of various aetiologies ${ }^{17-}$ 19 , and increased blood GFAP levels were also found in patients with $A D$ in comparison to healthy controls $^{20,21}$. Furthermore, an association between plasma GFAP levels and amyloid load in AD patients has been recently observed ${ }^{20-23}$, corroborating neuropathological findings.

The aim of this study was to examine GFAP and NfL levels in CSF and plasma in various stages of the clinical AD continuum compared to healthy controls. Furthermore, we investigated the predictive value of these blood biomarkers in combination with well-established risk factors in relation to clinical diagnosis and amyloid positivity in an outpatient memory clinic setting,

\section{Methods}

\section{Study population}

185 patients were enrolled in this retrospective study at the Memory Clinic of the Department of Neurology, Medical University of Vienna. Using two existing registries, i.e. the Dementia Registry RDA MUV (EK 1323/2018) and the BIOBANK MUV (EK 2195/2016), we identified 141 patients with a diagnosis along the clinical spectrum of cognitive decline, i.e. SCD $(n=18), \operatorname{MCl}(n=63)$ and $\operatorname{AD}(n=60)$. Additionally, 44 age-matched healthy controls $(\mathrm{HC})$ without signs of neurodegenerative disorder or cognitive decline were included. 
All 141 patients with cognitive complaints (SCD, MCl, AD) underwent a thorough standardized diagnostic examination including physical and neurological evaluation, neuropsychological testing, magnetic resonance imaging (MRI) of the brain and basic laboratory testing. For a subset of patients, we extended our diagnosis with a biomarker-based approach. CSF analysis of established AD biomarkers were available (e.g. Ab42, tTau, pTau) in 75 patients, amyloid-PET imaging was performed in 75 patients, and 54 patients underwent both diagnostic methods.

Diagnosis of $\mathrm{AD}$ and $\mathrm{MCl}$ were based on the recommendation of the National Institute of Ageing and Alzheimer's Association (NIA-AA $)^{4,24}$. The diagnosis of SCD was applied when no abnormalities on cognitive tests were observed and diagnosis criteria for $\mathrm{MCl}, \mathrm{AD}$ or other major neurological or psychiatric disorders were not met $^{25,26}$.

All 185 study participants were required to have a plasma EDTA sample stored in the Biobank MUV, for 103 study participants CSF samples were available as well.

The project was approved by the Ethics Committee of the Medical University of Vienna (EK 1965/2019) on November 28th, 2019.

\section{Neuropsychological assessment}

The Neuropsychological Test Battery Vienna (NTBV) was administered to assess cognitive function, including domains of attention, language, executive functioning and episodic memory and is described elsewhere ${ }^{27,28}$. Adequate normative data from cognitively unimpaired individuals were available and zscores for each variable were calculated, corrected for age, education and sex. Global cognition was computed by Mini Mental State Examination (MMSE) and Wortschatztest (WST), a standardized vocabulary test providing an estimate of premorbid IQ. Depressive symptoms were measured via Beck Depression Inventory (BDI-II) ${ }^{29}$.

\section{MR Imaging}

All patients underwent at least a T1-weighted MR sequence, a T2-weighted or a Fluid-attenuated inversion recovery (FLAIR) MR sequence and a diffusion-weighted MR sequence within the routine diagnostic setting for the evaluation of the extent and pattern of atrophy, the presence and degree of vascular lesions and to exclude other underlying pathologies causing cognitive decline (e.g. normal pressure hydrocephalus, subdural hematomas or brain tumors) and diffusion restricted areas (representing acute ischemia, inflammation or signal changes indicating Creutzfeldt-Jakob disease).

\section{Amyloid-PET Imaging}

75 patients underwent an Amlyoid-PET scan with [ $\left.{ }^{18} \mathrm{~F}\right]$ flutemetamol $(n=25)$ or $\left[{ }^{11} \mathrm{C}\right]$ Pittsburgh compound-B ( $\mathrm{PiB}, \mathrm{n}=50$ ). Amyloid PET imaging was performed on one of two possible PET scanner systems (Siemens Biograph 64 True Point, Erlangen, Germany or GE Advances PET, GE Healthcare Institute, Waukesha, Wisconsin, USA) belonging to the Division of Nuclear Medicine of the Medical University of Vienna. All studies were performed under strictly controlled conditions. Either $\sim 400 \mathrm{MBq}$ of 
[11C]PiB (in house production according to previously published recommendations ${ }^{30}$ ) or $185 \mathrm{MBq}$ of [18F]flutemetamol (Vizamyl®, GE Healthcare) were injected intravenously into a peripheral vein with starting image acquisition 40 minutes p.i. for [11C]PiB and 90 minutes p.i. for Vizamyl® for 20 minutes, where the tracer accumulation in the brain is reaching the maximum. Using the GE Advances PET scanner, a 3D ordered subset expectation maximization (OSEM) filtered backprojection (FBP) reconstruction was done (128x128 matrix, Hanning filer, cut-off $6.2 \mathrm{~mm})$. For [18F]flutemetamol, using the Siemens Biograph 64 True Point the reconstruction was performed using a 3D OSEM with 4 iterations and 21 subsets into a $168 \times 168$ matrix with a Zoom of $2(2 \times 2 \mathrm{~mm}$ pixel size), and with a $5 \mathrm{~mm}$ full width at half-maximum (FWHM) Hann post reconstruction filter applied to the final images. Subsequently, the image acquisition was performed for about 20 min following a computed tomography (CT) acquisition for attenuation correction using Siemens Biograph 64 True Point.

APOE genotyping

Genomic DNA was extracted from peripheral blood leucocytes of individuals with available whole blood samples $(n=159)$ using standard DNA isolation methods. Apolipoprotein $E(A P O E)$ genotyping was performed using quantitative polymerase chain reaction (qPCR) with TaqMan probes (Thermofisher) evaluating two single nucleotide polymorphisms (SNPs) in the APOE gene (rs429358 and rs7412). Each sample was tested for both SNPs in triplicates using $20 \mathrm{ng}$ DNA. Allelic discrimination analysis was used to determine the $A P O E$ genotype of the study participants.

\section{CSF biomarkers}

CSF was obtained by lumbar puncture between the L3/L4, L4/5 or L5/S1 intervertebral space, collected in polypropylene tubes and further stored at $-20^{\circ} \mathrm{C}$ until biomarker analysis (as for Ab42, pTau 181 and tTau), or immediately at $-80^{\circ} \mathrm{C}$ for future research purposes ${ }^{31,32}$. Levels of Ab42, pTau 181 and tTau were measured with commercially available enzyme-linked immunosorbent assays (ELISA) (Innotest hTAU-Ag,

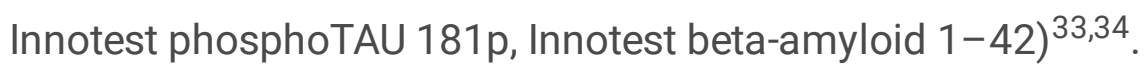

\section{Plasma biomarkers}

EDTA plasma was collected through venepuncture and stored at $-80^{\circ} \mathrm{C}$ in our local biobank.

Concentrations of NfL and GFAP were quantified with ultrasensitive single molecule array (SIMOA) using the Neurology 2-Plex B kit from Quanterix in CSF and plasma. Detailed analyses are described

elsewhere ${ }^{35}$. In short, equilibrated calibrators, samples and controls were diluted (1:4 for plasma and 1:100 for CSF) and incubated with detector and paramagnetic reagents provided by the manufacturer. Streptavidin ß-galactosidase was added to each well before samples were transferred to the Quanterix SR-X analyser for measurement of protein levels. All samples were analysed as duplicates and all assay materials were obtained from the same kit lot. Intra-assay CV was $<12 \%$ for GFAP and $<8 \%$ for NfL. Interassay CV for two samples measured repeatedly on 10 plates was well acceptable ( $<13 \%$ for GFAP and $9 \%$ for $N f L$ ).

\section{Statistical Analysis}


Data is presented as $\mathrm{n}$ (percent) or median (interquartile range) as appropriate. Testing for differences between groups was performed using chi-square test, the Mann-Whitney-U-test or the Kruskal-Wallis test. Correlation was assessed using Spearman's rank correlation coefficient. To evaluate the discriminatory performance of the biomarkers assessed herein, the cohort was split into pairs of two diagnosis (e.g. AD and $\mathrm{HC}$ ) and the response variable was coded as existing for the more severe diagnosis (i.e. SCD when assessing SCD vs $\mathrm{HC}$ ). Next, a baseline model consisting of sex, age and APOE4 status (increased risk for carriers of the APOE4 allele) was constructed using logistic regression. A receiver operating characteristic (ROC) curve was plotted and the area under the curve (AUC) was measured. Optimal cutoffs were calculated using Youden's J-Statistic ${ }^{36}$ and sensitivity and specificity are reported as percentage. The baseline model was then supplemented by either levels of plasma GFAP, plasma NfL, or both and the AUC of each model was compared using DeLong's test for correlated AUC curves ${ }^{37}$. A p value of $<0.05$ was interpreted as statistically significant. All calculations were performed in $\mathrm{R}$ (Version 4.0.4) and the pROC package was used for ROC calculations ${ }^{38}$

\section{Results}


Table 1

Demographics and clinical characteristics

\begin{tabular}{|c|c|c|c|c|c|}
\hline & $\mathrm{HC}$ & SCD & $\mathrm{MCl}$ & $A D$ & $p$ value \\
\hline $\mathbf{N}$ & 44 & 18 & 63 & 60 & \\
\hline $\operatorname{Sex}(f)$ & $24(54.5 \%)$ & $\begin{array}{l}8 \\
(44.4 \%)\end{array}$ & $\begin{array}{l}29 \\
(46.0 \%)\end{array}$ & $36(60.0 \%)$ & $p=0.399$ over all groups \\
\hline Age & $\begin{array}{l}61.2 \\
(55.8,69.5)\end{array}$ & $\begin{array}{l}68.3 \\
(60.1 \\
77.9)\end{array}$ & $\begin{array}{l}69.9 \\
(59.3 \\
77.8)\end{array}$ & $\begin{array}{l}69.0(61.4 \\
75.1)\end{array}$ & $\begin{array}{l}p<0.05 \text { for } \mathrm{HC} \text { vs. SCD, } p<0.01 \\
\text { for } \mathrm{HC} \text { vs. } \mathrm{MCl} \text { and } \mathrm{AD}\end{array}$ \\
\hline MMSE & n.a. & $\begin{array}{l}29(28, \\
30)\end{array}$ & $\begin{array}{l}27(25 \\
28)\end{array}$ & $20(14,23)$ & $\begin{array}{l}p<0.001 \text { for AD vs. SCD and } \mathrm{MCl} \text {, } \\
p<0.01 \text { for SCD vs. } \mathrm{MCl}\end{array}$ \\
\hline $\begin{array}{l}\text { APOE4 } \\
\text { carrier }\end{array}$ & $12(33.3 \%)$ & $\begin{array}{l}3 \\
(18.8 \%)\end{array}$ & $\begin{array}{l}22 \\
(40.7 \%)\end{array}$ & $33(62.3 \%)$ & $\mathrm{p}<0.01$ over all groups \\
\hline $\begin{array}{l}\text { CSF Ab42 } \\
(\mathrm{pg} / \mathrm{ml})\end{array}$ & n.a. & n.a. & $\begin{array}{l}354 \\
(248, \\
479.5)\end{array}$ & $\begin{array}{l}332.5 \\
(231.8 \\
454.8)\end{array}$ & $p=0.370$ for $\mathrm{MCl}$ vs. $A D$ \\
\hline $\begin{array}{l}\text { CSF tTau } \\
\text { (pg/ml) }\end{array}$ & n.a. & n.a. & $\begin{array}{l}310 \\
(188, \\
504.5)\end{array}$ & $\begin{array}{l}600.5 \\
(404.3 \\
1106.8)\end{array}$ & $p<0.001$ for $\mathrm{MCl}$ vs. $\mathrm{AD}$ \\
\hline $\begin{array}{l}\text { CSF pTau } \\
\text { (pg/ml) }\end{array}$ & n.a. & n.a. & $\begin{array}{l}53 \\
(33.5 \\
79.5)\end{array}$ & $\begin{array}{l}77.5(51.3 \\
96.3)\end{array}$ & $p<0.05$ for $\mathrm{MCl}$ vs $\mathrm{AD}$ \\
\hline $\begin{array}{l}\text { Positive } \\
\text { amyloid } \\
\text { PET }\end{array}$ & n.a. & n.a. & $\begin{array}{l}20 \\
(55.6 \%)\end{array}$ & 37 (94.9\%) & $\mathrm{p}<0.001$ for $\mathrm{MCl}$ vs. $A D$ \\
\hline \multicolumn{6}{|c|}{$\begin{array}{l}\text { Data is presented as median and interquartile range (IQR, } 25 \text { th }-75 \text { th percentile) or } \mathrm{n}(\%) \text {. } \\
\text { Demographic and clinical differences were measured using Kruskal-Wallis test or chi-square tests as } \\
\text { appropriate. APOE status was available for } 159 \text { patients }(36 \mathrm{HC}, 16 \mathrm{SCD}, 54 \mathrm{MCl}, 53 \mathrm{AD}) \text {, CSF AD } \\
\text { biomarkers (Ab42, tTau, pTau) for } 75 \text { patients ( } 37 \mathrm{MCl}, 38 \mathrm{AD}) \text { and amyloid-PET for } 75 \text { patients ( } 36 \\
\mathrm{MCl}, 39 \mathrm{AD}) \text {. }\end{array}$} \\
\hline \multicolumn{6}{|c|}{$\begin{array}{l}H C \text { healthy control, } S C D \text { subjective cognitive decline, } M C / \text { mild cognitive impairment, } A D \text { Alzheimer's } \\
\text { disease, } f \text { female, } M M S E \text { mini mental state examination, } C S F \text { cerebrospinal fluid, } A b 42 \text { amyloid-beta } \\
42, t T a u \text { total tau, } p T a u \text { phosphorylated tau, } n . a \text {. not available }\end{array}$} \\
\hline
\end{tabular}

Demographic and clinical characteristics are listed in Table 1.

We observed no significant difference in sex distribution between the groups $(p=0.399)$.

$\mathrm{HC}$ were significantly younger than the patient groups ( $p<0.05$ for $\mathrm{HC}$ vs. SCD, $\mathrm{p}<0.01$ for $\mathrm{HC}$ vs. $\mathrm{MCl}$ and $A D$ ), but there was no difference in age between the three different patient cohorts. MMSE decreased significantly with progressing disease with the lowest score in the AD group $(p<0.001$ for AD vs. SCD and $\mathrm{MCl}, \mathrm{p}<0.01$ for SCD vs. MCl). 
Data of $A P O E 4$ carriership (carriers of at least one APOE4 allele) was available for 159 patients, i.e. in 36 controls, 16 patients with $\mathrm{SCD}, 54$ with $\mathrm{MCl}$ and 53 with $\mathrm{AD}$, with the highest occurrence of $A P O E 4$ alleles in $A D$ patients (33 of 53 patients, $62.3 \%$ ). A chi-square test of independence was performed to examine the relation between the $A P O E 4$ status and the diagnosis. As can be seen by the frequencies cross tabulated in Table 1, there was a significant relationship between APOE4 status and diagnosis $\left(X^{2}(3, N=\right.$ $185)=13.21, p<0.01)$.

For a subset of patients $(n=75)$ CSF analysis of established AD biomarkers were available (e.g. Ab42, tTau, pTau). Regarding the concentration of the AD biomarkers, median levels of tTau in $\mathrm{MCl}$ and $A D$ were $310 \mathrm{pg} / \mathrm{ml}($ IQR 188, 504.5) and 600.5pg/ml (IQR 404.3, 1106.8), respectively, pTau $53 \mathrm{pg} / \mathrm{ml}$ (IQR 33.5, $79.5)$ and $77.5 \mathrm{pg} / \mathrm{ml}($ IQR $51.3,96.3)$, respectively and amyloid-beta $354 \mathrm{pg} / \mathrm{ml}($ IQR $248,479.5)$ and $332.5 \mathrm{pg} / \mathrm{ml}(231.8,454.8)$, respectively. While CSF tTau and pTau levels increased significantly with progression from $\mathrm{MCl}$ to $\mathrm{AD}$ ( $\mathrm{p}<0.001$ for $\mathrm{MCl}$ vs. $A D$ for tTau and $\mathrm{p}<0.05$ for $\mathrm{MCl}$ vs. $A D$ for $\mathrm{pTau}$ ), the difference of $A b 42$ concentration between $\mathrm{MCl}$ and $A D$ reached no statistical significance $(p=0.370$ for $\mathrm{MCl}$ vs. $\mathrm{AD}$ ).

Amyloid-PET imaging was performed in 36 patients with $\mathrm{MCl}$ and 39 patients with $\mathrm{AD}$. Amyloid-PET detected the cerebral cortical accumulation of amyloid-beta in $20 \mathrm{MCl}$ patients (55.6\%). Positive amyloid PET imaging was significantly higher in AD patients with a total of $37(94.9 \%)$ positive subjects $(p<0.001$ for $\mathrm{MCl}$ vs. $\mathrm{AD}$ ). 
Table 2

Plasma and CSF Results for NfL and GFAP

\begin{tabular}{|c|c|c|c|c|c|}
\hline & $\mathrm{HC}$ & SCD & $\mathrm{MCl}$ & $A D$ & $p$ value \\
\hline$N$ & 44 & 18 & 63 & 60 & \\
\hline $\begin{array}{l}\text { Plasma } \\
\text { NFL } \\
\text { (pg/ml) }\end{array}$ & $\begin{array}{l}8.1(5.9 \\
12.2)\end{array}$ & $\begin{array}{l}15.8 \\
(8.2 \\
18.6)\end{array}$ & $\begin{array}{l}\text { 12. } 9 \\
20.4)\end{array}$ & $\begin{array}{l}15.5(11.8 \\
23.2)\end{array}$ & $\begin{array}{l}p<0.001 \text { for } \mathrm{HC} \text { vs. } \mathrm{MCl} \text { and } \mathrm{AD}, p< \\
0.01 \text { for } \mathrm{HC} \text { vs. } S C D, p<0.05 \text { for } \mathrm{MCl} \\
\text { vs } \mathrm{AD}\end{array}$ \\
\hline $\begin{array}{l}\text { Plasma } \\
\text { GFAP } \\
\text { (pg/ml) }\end{array}$ & $\begin{array}{l}79.0(53.7 \\
120.6)\end{array}$ & $\begin{array}{l}111.0 \\
(71.0 \\
154.0)\end{array}$ & $\begin{array}{l}167.5 \\
(93.8 \\
256.3)\end{array}$ & $\begin{array}{l}181.9 \\
(129.6 \\
269.6)\end{array}$ & $\begin{array}{l}p<0.001 \text { for } \mathrm{HC} \text { vs. } \mathrm{MCl} \text { and } \mathrm{AD}, p< \\
0.01 \text { for } \mathrm{SCD} \text { vs. } A D, p<0.05 \text { for SCD } \\
\text { vs. MCl }\end{array}$ \\
\hline $\mathbf{N}$ & 36 & 0 & 30 & 37 & \\
\hline $\begin{array}{l}\text { CSF } \\
\text { NFL } \\
(\mathrm{pg} / \mathrm{ml})\end{array}$ & $\begin{array}{l}584.1 \\
(449.6 \\
832.8)\end{array}$ & n.a. & $\begin{array}{l}807.7 \\
(507.6 \\
1103.2)\end{array}$ & $\begin{array}{l}1559.1 \\
(1026.6 \\
2513.9)\end{array}$ & $\begin{array}{l}p<0.001 \text { for } \mathrm{HC} \text { vs. } A D \text { and } \mathrm{MCl} \text { vs. } \\
\mathrm{AD}\end{array}$ \\
\hline $\begin{array}{l}\text { CSF } \\
\text { GFAP } \\
(\mathrm{pg} / \mathrm{ml})\end{array}$ & $\begin{array}{l}11145.3 \\
(6980.5 \\
14373.8)\end{array}$ & n.a. & $\begin{array}{l}8946.2 \\
(7028.8 \\
13842.7)\end{array}$ & $\begin{array}{l}13663.5 \\
(9945.4 \\
21059.1)\end{array}$ & $\begin{array}{l}p<0.01 \text { for } M C l \text { vs. } A D, p<0.05 \text { for } \\
\text { HC vs. } A D\end{array}$ \\
\hline
\end{tabular}

Data is presented as median and interquartile range (IQR, 25th -75 th percentile) or $\mathrm{n}$. Differences of biomarker concentration were calculated using Kruskal-Wallis test. CSF data for GFAP and NFL were available for 103 patients.

$H C$ healthy control, $S C D$ subjective cognitive decline, $M C /$ mild cognitive impairment, $A D$ Alzheimer's disease, $C S F$ cerebrospinal fluid, $N f L$ neurofilament light chain, GFAP glial fibrillary acidic protein, $n . a$. not available

Plasma GFAP showed a gradual increase along the four cohorts, with the lowest concentration in HC (median $79 \mathrm{pg} / \mathrm{ml}$, IQR 53.7, 120.6) and the highest in patients with AD (median 181.9pg/ml, IQR 129.6, 269.6) (Table 2). Besides significantly higher levels of plasma GFAP in patients with $\mathrm{MCl}$ and AD compared to healthy controls (Fig. 1, $p<0.001$ ), we observed a significant difference between patients with $S C D$ and $A D(p<0.01)$ and patients with SCD and $\mathrm{MCl}(p<0.05)$. The difference between $\mathrm{HC}$ and $S C D$, as well as between $\mathrm{MCl}$ and $A D$ missed statistical significance $(p=0.092$ and $p$ $=0.098$, respectively).

Plasma NfL performed similar to GFAP regarding the difference in concentrations between $\mathrm{HC}$ and $\mathrm{MCl} / \mathrm{AD}(\mathrm{p}<0.001$, Table 2 and Fig. 1). However, NfL levels showed a significant difference between $\mathrm{HC}$ and SCD $(p<0.01)$ as well as between $\mathrm{MCl}$ and $\mathrm{AD}(p<0.05)$, but not between SCD and MCl/AD. Interestingly, we found the highest concentration of NfL in patients with SCD (median $15.8 \mathrm{pg} / \mathrm{ml}$, IQR 8.2, 18.6).

For 103 patients, CSF samples in our local biobank were available. While levels of CSF NfL increased gradually (HC 584.1 pg/ml IQR 449.6, 832.8; MCl 807.7pg/ml IQR 507.6, 1103.2; AD 1559.1 pg/ml IQR 1026.6, 2513.9), CSF GFAP presented the lowest concentration in the $\mathrm{MCl}$ group $(8.946 .2 \mathrm{pg} / \mathrm{ml}$ IQR 7028.8, 13842.7), followed by HC (11.145.3pg/ml IQR 6980.5, 14373.8) and AD (13.663.5pg/ml IQR 9945.4, 21059.1). Concerning the performance of these two CSF biomarkers in distinguishing between $\mathrm{HC}$ and $\mathrm{AD}$ or $\mathrm{MCl}$ and $\mathrm{AD}, \mathrm{CSF} \mathrm{NfL}$ showed slightly better results $(\mathrm{p}<0.001)$ in comparison to CSF GFAP ( $p<0.05$ and $p<0.01$, respectively).

Using Spearman correlation coefficient, the correlation of NfL and GFAP in CSF and plasma were analysed (Fig. 2a and 2b). Correlation between NfL in CSF and plasma was calculated with R $=0.64$ ( $p<$ 
0.001, Fig. 2a) and GFAP in CSF and plasma with $R=0.4$ ( $p<0.001$, Fig. $2 b)$.

To assess the clinical utility of GFAP and NfL in plasma, particularly in distinguishing healthy controls from patients with cognitive complaints (e.g. $S C D, M C l$ and $A D$ ) and potentially predicting cerebral amyloid status as measured by amyloid-PET imaging, ROC analyses were performed and adjusted for sex and age. We constructed a diagnostic panel, consisting of well - established risk factors such as age, sex (defined as female $>$ male), and APOE4 carriership (defined as carrying at least one copy of the $A P O E 4$ allele) (i.e. age + sex $+A P O E 4$ panel) and compared it with a panel of age, sex, APOE4 carriership added by plasma NfL and plasma GFAP, called age + sex + APOE4 + GFAP + NfL panel (Fig. 3a-g). Additionally, we analysed in this model each biomarker separately to assess the potential benefit of GFAP or NfL alone (i.e. age + sex + APOE4 + GFAP panel and age + sex + APOE4 + NfL panel).

The age + sex + APOE4 + GFAP + NfL panel performed better in discriminating HC from AD (AUC 91.6\%) than $\mathrm{HC}$ from $\mathrm{MCl}$ (AUC 81.7\%) and outperformed the age + sex + APOE4 panel alone $(p<0.001$ and $p=$ 0.004 , respectively) (Fig. $3 \mathrm{a}$ and $3 \mathrm{~b}$ ). When assessing each biomarker separately, the age + sex $+A P O E 4+$ GFAP panel outperformed the age + sex + APOE4 + NfL panel in distinguishing $\mathrm{HC}$ from $\mathrm{MCl}$ and $\mathrm{AD}$. The age + sex + APOE4 + GFAP panel distinguished $\mathrm{HC}$ from $\mathrm{MCl}$ and $\mathrm{AD}$ with an $\mathrm{AUC}$ of $81.3 \%, \mathrm{p}=0.005$ and an AUC of $91.3 \%, \mathrm{p}<0.001$, respectively, compared to the age + sex $+A P O E 4$ panel, whereas the age + sex + APOE4 + NfL panel showed no additional benefit in differentiating $\mathrm{HC}$ from $\mathrm{MCl}$ (AUC 68.8\%, $\mathrm{p}=$ $0.2936)$, and differentiating $\mathrm{HC}$ vs. AD was inferior to the age + sex + APOE4 + GFAP panel and the age + sex + APOE4 + GFAP + NfL panel (AUC 84.5\%, $\mathrm{p}=0.003)$.

When comparing patients with $\mathrm{MCl}$ and $\mathrm{AD}$, the age + sex + APOE4 + GFAP + NfL panel (AUC 72.3\%) and the age + sex $+A P O E 4+\mathrm{NfL}$ panel (AUC 72\%) performed slightly better than the age + sex + APOE4 panel (AUC 66.4\%) and the age + sex + APOE4 + GFAP panel (AUC 66.7\%), but no significant difference was observed (Fig. 3c).

In terms of distinguishing SCD from the other cohorts, the age + sex + APOE4 + GFAP + NfL panel outperformed the age + sex $+A P O E 4$ panel significantly $(p<0.05)$. The best results were obtained for SCD vs. AD (AUC 85\%, Fig. 3d), followed by SCD vs. MCl (AUC 81.3\%, Fig. 3e) and SCD vs. HC (77.7\%, Fig. 3f). Again, adding GFAP alone outperformed adding NfL alone. The age + sex + APOE4 + NfL panel showed no significant improvement compared to the age + sex + APOE4 panel (SCD vs. AD AUC 78.8\%, SCD vs. MCl AUC $64.7 \%$, SCD vs. HC AUC 73.8\%), while the age + sex + APOE4 + GFAP panel demonstrated a significantly higher AUC compared to the age + sex + APOE4 panel for SCD vs. AD $(84.7 \%, p<0.05)$, whereas the distinction between SCD vs. MCI (AUC 74.4\%) and SCD. vs HC (AUC 76.4\%) missed statistical significance.

Regarding the diagnostic accuracy in predicting amyloid-PET status and the distinction of amyloidnegative (Ab-) from amyloid-positive $(\mathrm{Ab}+)$ individuals, AUC for the age + sex $+A P O E 4+\mathrm{GFAP}+\mathrm{Nfl}$ panel was calculated with $88.4 \%$ and was therefore superior than the age + sex $+A P O E 4$ panel alone (AUC 75\%, $\mathrm{p}<0.05$, Fig. 3g). The age + sex + APOE4 + GFAP panel obtained an AUC of $86.9 \%$ but missed statistical 
significance compared to the age + sex $+A P O E 4$ panel alone $(p=0.07)$. Results for age + sex $+A P O E 4+$ $\mathrm{NfL}$ were similar to the age + sex + APOE4 panel (AUC 77.4\%).

\section{Discussion}

In this outpatient memory clinic-based study we examined the performance of two promising biomarkers of neurodegeneration and neuroinflammation, e.g. NfL and GFAP, for the diagnostic work-up of patients along the continuum of AD-related cognitive decline. We aimed to develop a practical and reproducible model for a quick and accurate patient diagnosis in an easy-to-handle way for an outpatient memory clinic setting. As age, sex and APOE4 status are the most well-known risk factors for $A D{ }^{39,40}$ and are already part of an established diagnostic work - up in many memory clinic settings, we contemplated an extended risk model that included these risk factors in addition to plasma GFAP and NfL levels (age + sex $+A P O E 4+\mathrm{GFAP}+\mathrm{NfL}$ panel). To assess the best performance of each single biomarker, we furthermore analysed the impact of adding GFAP (age + sex + APOE4 + GFAP panel) or NfL alone (age + sex + APOE4 + NfL panel).

The combined age + sex $+A P O E 4+\mathrm{GFAP}+\mathrm{NfL}$ panel could differentiate healthy controls from patients with $\mathrm{MCl}$ or $A D$ and significantly outperformed the age + sex $+A P O E 4$ panel alone. For discriminating the disease states of $\mathrm{MCl}$ and $\mathrm{AD}$, the age $+\mathrm{sex}+A P O E 4+\mathrm{GFAP}+\mathrm{NfL}$ panel could not add a significant benefit to the age + sex + APOE4 panel.

We further investigated the potential of the age + sex + APOE4 + GFAP + NfL panel in regard of discriminating patients with SCD from the other cohorts and found promising results, especially in differentiating SCD from patients with an objective cognitive decline $(\mathrm{MCl}$ and $A D)$, representing therefore a potential useful complementary diagnostic tool in an outpatient setting. In terms of predicting amyloid positivity on PET-imaging the age + sex + APOE4 + GFAP + NfL panel performed significantly better than the age + sex $+A P O E 4$ panel alone and showed a good distinction between $A b+$ and $A b$-patients. When analysing the performance of a combination of each biomarker alone, the age + sex + APOE4 + GFAP panel outperformed the age + sex $+A P O E 4+\mathrm{NfL}$ panel in all examined patient cohorts, except for $\mathrm{MCl} v \mathrm{v}$. $A D$.

Focusing on plasma GFAP alone, its levels showed a gradual increase along the four cohorts, with the lowest concentration in $\mathrm{HC}$ and the highest in patients with $\mathrm{AD}$, thereby allowing a good biological interpretation of a gradual rise of this biomarker along the progressing neuropathological process. Plasma GFAP alone achieved the most prominent discrimination between $\mathrm{HC}$ and patients with an objective cognitive decline ( $\mathrm{MCl}$ and $\mathrm{AD}$ ). Moreover, the SCD group could be discriminated significantly from $\mathrm{MCl}$ and $A D$, however, $\mathrm{MCl}$ and $A D$ as well as $H C$ and $S C D$ could not be differentiated by the use of plasma GFAP alone.

Plasma NfL alone could best discriminate between $\mathrm{HC}$ and $\mathrm{MCl} / \mathrm{AD}$ patients and showed also promising demarcation between the $\mathrm{HC}$ and $\mathrm{SCD}$ group and even more interesting between the $\mathrm{MCl}$ and $\mathrm{AD}$ group. 
Intriguingly, we found the highest concentration of plasma NfL in patients with SCD, which might not be solely explained by the small sample size. Therefore, these results must be interpreted cautiously.

Diagnosis of early phases of $A D$ is crucial in regard of detecting patients at-risk as early as possible in the development of the neuropathological cascade. Besides the abnormal aggregation of Ab peptide and tau protein, neuroinflammation and neurodegeneration represent major components in the pathophysiology of $A D^{4}$. Due to the recent evolvement of more sensitive analytical methods, non-invasive blood-based biomarkers could serve as a reliable approximation to this early pathogenic process. In recent years, the role of neuroinflammation in the pathogenesis of $A D$ has been increasingly focused on in the literature. Neuropathological data have shown a close spatial relationship between Ab plaques and reactive astrocytes, which along with microglia, may trigger a pro-inflammatory cascade and eventually lead to neurodegeneration, which in turn activates astrocytes and microglia ${ }^{41,42}$. As a cytoskeletal component of astrocytes, GFAP could serve as a promising biomarker reflecting astrocytic activation and proliferation during the neurodegenerative processes, including $A D$, particularly in its earliest stages ${ }^{43,44}$. On the other hand, $\mathrm{NfL}$ represents a rather unspecific biomarker for neurodegeneration, as it is released by axonal damage in multiple neurological disorders ${ }^{45,46}$. While the importance of $\mathrm{NfL}$ as a blood-based biomarker has been already reported in several studies ${ }^{12,13,47,48}$, the significance of GFAP is currently still evolving. To our knowledge, only few studies have evaluated the combination of GFAP with other biomarkers so far, and presented the utility of plasma GFAP not just in discriminating healthy controls from patients with $A D$ but also in distinguishing $A b+$ from $A b$-individuals ${ }^{21-23}$. Furthermore, higher GFAP levels have been associated with an increased risk for future progression to dementia and a steeper cognitive decline 43,49 . Our data encourage the application of a blood-based biomarker model for an outpatient memory clinic setting, with even more promising results for GFAP than NfL for the discrimination of early stages along the AD continuum, whereas NfL might be more helpful in discriminating later stages. This could be in line with data, in which NfL has been reported to be rather a marker for progression of disease, whereas GFAP could be superior in identifying patients at-risk for developing dementia ${ }^{43}$. Our data may support this hypothesis. Regarding the heterogeneity of AD pathology, a panel of well-combined blood-based biomarkers could aid in the early detection as well as disease monitoring in the future. The failure to show a discriminatory superiority of the age + sex $+A P O E 4+\mathrm{GFAP}+\mathrm{NfL}$ panel in regard of differentiation of $\mathrm{MCl}$ and $\mathrm{AD}$, while showing a slightly better performance of plasma $\mathrm{NfL}$ in this matter, might further underline this concept.

While recent studies increasingly focus on the evaluation of biomarkers in blood, especially in terms of feasibility, CSF biomarkers in AD have partially faded from the spotlight. In our cohort, we found the highest concentration of CSF GFAP in patients with AD followed by the HC group and the lowest concentration in $\mathrm{MCl}$.

CSF NfL demonstrated a gradual increase over the three cohorts with the lowest levels in $\mathrm{HC}$ and the highest in AD and concentration of NfL in CSF and plasma correlated well with each other, which is in line 
with already published data 9,12,50-52, suggesting that plasma levels might be considered as an acceptable proxy of CSF levels.

We found the lowest concentration of CSF GFAP in patients with $\mathrm{MCl}$, followed by the $\mathrm{HC}$ and $\mathrm{AD}$ group, therefore results must be interpreted cautiously. In contrast to NfL, levels of GFAP in CSF and plasma showed a lower correlation, which was already described by another study ${ }^{21}$. While other studies analysed levels of GFAP with ELISA ${ }^{18,19,21,53}$, we found no comparable results for measurement of CSF GFAP with SIMOA $\rightarrow$ technology in patients along the AD continuum. Thus, further investigations are needed to better determine the role of CSF GFAP and its correlation with GFAP levels in blood in these patient cohorts.

Nevertheless, both measurements- CSF GFAP and CSF NfL- allowed a good discrimination between HC and $A D$ as well as $M C l$ and $A D$, with better results for CSF NfL.

\section{Limitations}

A limitation of our study is its retrospective and cross-sectional nature. Therefore, we lack longitudinal follow-up results and miss data in some of the patients. Positive amyloid-PET and APOE4 carriership were significantly less common in the $\mathrm{MCl}$ group, which might lead to the notion, that at least some of the $\mathrm{MCl}$ patients are not along the AD continuum. Neuropathological data could not be attained in our in vivo patient cohort. The fact that the highest concentration of plasma NfL was found in patients with SCD could be interpreted as a sign of inclusion of a too diverse population, caused by the known heterogeneity of this diagnostic entity. Still, since we focused on patients with putative AD pathology, we excluded patients with other causes of dementia or high vascular burden, which led to a smaller sample size. Moreover, healthy controls were significantly younger than the patient groups, which might influence results of these biomarkers and corresponding analysis. To counteract this potential bias in our data, ROC analyses were adjusted for sex and age.

\section{Conclusion}

Blood-based biomarkers for AD may represent a valuable complementary tool for the clinical diagnosis and patient management in the near future. As to the increasing incidence of patients suffering from neurodegenerative disorders, developing minimally invasive and affordable biomarkers might confer great benefit in a quick and accurate diagnostic work -up. The outstanding benefits of blood-based biomarkers are the good availability and the potential of repetitive analysis in an individual patient without major efforts. In a time, where clinical trials are increasingly focusing on early stages of $A D$, it is crucial to develop a reliable diagnostic method that can be used as an easy-to-apply screening tool. We suggest that plasma GFAP could aid in a better distinction of patients along different predementia stages and that the combination of GFAP and NfL plasma levels with conventional risk factors could serve as a good "at-risk" model for early patient selection and assessment of progression to AD. Regarding the 
heterogeneity of $A D$ pathology, the implementation of a panel of well combined blood-based biomarkers could serve as a valuable adjunct during the diagnostic process in an outpatient memory clinic setting.

\section{Abbreviations}

$A b$

$A D$

APOE

AUC

BDI-II

CSF

CT

e.g.

ELISA

f

FBP

FLAIR

FWHM Full width at half-maximum

GFAP Glial Fibrillary Acidic Protein

HC Healthy control

i.e. id est

IQR Interquartile range

$\mathrm{MCl} \quad$ Mild cognitive impairment

MMSE Mini-Mental State Examination

MRI Magnetic resonance imaging

MUV Medical University of Vienna 


$\begin{array}{ll}\text { n.a. } & \text { not available } \\ \text { NfL } & \text { Neurofilament light chain } \\ \text { NTBV } & \text { Neuropsychological Test Battery Vienna } \\ \text { NFT } & \text { Neurofibrillary tangles } \\ \text { NIA-AA } & \text { National Institute on Aging and Alzheimer's Association } \\ \text { ns } & \text { Not significant } \\ \text { OSEM } & \text { Ordered subset expectation maximization } \\ \text { PET } & \text { Positron emissions tomography } \\ \text { PiB } & \text { Pittsburgh Compound B } \\ \text { pTau } & \text { Phosphorylated Tau } \\ \text { qPCR } & \text { Quantitative polymerase chain reaction } \\ \text { RDA } & \text { Research Documentation and Analysis } \\ \text { ROC } & \text { Receiver operating characteristic } \\ \text { SCD } & \text { Subjective cognitive decline } \\ \text { SIMOA } & \text { Ultrasensitive single-molecule array } \\ \text { SNP } & \text { Single nucleotide polymorphisms } \\ \text { tTau } & \text { Total Tau } \\ \text { WST } & \text { Wortschatztest } \\ & \\ \text { NT } & \end{array}$

\section{Declarations}

\section{Ethics approval and consent to participate}

The project was approved by the Ethics Committee of the Medical University of Vienna (EK 1965/2019) on November 28th, 2019 and renewed on November $27^{\text {th }}, 2020$. The study was conducted in accordance with the Helsinki Declaration.

Consent for publication

Not applicable 
Availability of data and materials

The datasets used and/or analysed during the current study are available from the corresponding author on reasonable request.

\section{Competing interests}

The authors declare that they have no competing interests

\section{Funding}

Not applicable.

\section{Author's contributions}

TP, RW and ES devised the protocol. TP collected and managed the data with contribution of RW, SS and ES. TP and TK performed SIMOA analyses in CSF and plasma. TP and RW performed the statistical analysis. TP and ES interpreted the data and prepared the manuscript. RW, TK, PR, SK, EG, PA, TTW and JF provided feedback and major contribution to the manuscript. All authors read and approved the final manuscript.

\section{$\underline{\text { Acknowledgements }}$}

We would like to thank the patients and their families whose help and participation made this work possible. In addition, we thank contributors who collected samples used in this study.

\section{References}

1. McKhann G, et al. Clinical diagnosis of Alzheimer's disease Report of the NINCDS-ADRDA Work Group* under the auspices of Department of Health and Human Services Task Force on Alzheimer's Disease. Neurology. 1984;34:939-9.

2. McKhann G, et al. Clinical diagnosis of Alzheimer's disease: Report of the NINCDS-ADRDA Work Group under the auspices of Department of Health and Human Services Task Force on Alzheimer's Disease. Neurology. 2011;77:333-3.

3. Bateman RJ, et al. Clinical and Biomarker Changes in Dominantly Inherited Alzheimer's Disease. New Engl J Medicine. 2012;367:795-804.

4. Jack CR, et al. NIA-AA Research Framework: Toward a biological definition of Alzheimer's disease. Alzheimer's Dementia. 2018;14:535-62.

5. Sperling RA, et al. Toward defining the preclinical stages of Alzheimer's disease: Recommendations from the National Institute on Aging-Alzheimer's Association workgroups on diagnostic guidelines for Alzheimer's disease. Alzheimer's Dementia. 2011;7:280-92. 
6. Terry RD, et al. Physical basis of cognitive alterations in alzheimer's disease: Synapse loss is the major correlate of cognitive impairment. Ann Neurol. 1991;30:572-80.

7. Moya-Alvarado G, Gershoni-Emek N, Perlson E, Bronfman FC. Neurodegeneration and Alzheimer's disease (AD). What Can Proteomics Tell Us About the Alzheimer's Brain?*. Mol Cell Proteomics. 2016;15:409-25.

8. Wilson DH, et al. The Simoa HD-1 Analyzer. Slas Technology. 2015;21:533-47.

9. Kuhle J, et al. Comparison of three analytical platforms for quantification of the neurofilament light chain in blood samples: ELISA, electrochemiluminescence immunoassay and Simoa. Clin Chem Laboratory Medicine Cclm. 2016;54:1655-61.

10. Bridel C, et al. Diagnostic Value of Cerebrospinal Fluid Neurofilament Light Protein in Neurology. Jama Neurol. 2019;76:1035-48.

11. Lewczuk P, et al. Plasma neurofilament light as a potential biomarker of neurodegeneration in Alzheimer's disease. Alzheimer's Res Ther. 2018;10:71.

12. Mattsson N, Andreasson U, Zetterberg H, Blennow K, Initiative A. D. N. Association of Plasma Neurofilament Light With Neurodegeneration in Patients With Alzheimer Disease. Jama Neurol. 2017;74:557.

13. Mattsson N, Cullen NC, Andreasson U, Zetterberg H, Blennow K. Association Between Longitudinal Plasma Neurofilament Light and Neurodegeneration in Patients With Alzheimer Disease. Jama Neurol. 2019;76:791-9.

14. Wolf $F$ de, et al. Plasma tau, neurofilament light chain and amyloid- $\beta$ levels and risk of dementia; a population-based cohort study. Brain. 2020;143:1220-32.

15. Verkhratsky A, Olabarria M, Noristani HN, Yeh C-Y, Rodriguez JJ. Astrocytes in Alzheimer's disease. Neurotherapeutics. 2010;7:399-412.

16. Kamphuis W, et al. Glial fibrillary acidic protein isoform expression in plaque related astrogliosis in Alzheimer's disease. Neurobiol Aging. 2014;35:492-510.

17. Colangelo AM, Alberghina L, Papa M. Astrogliosis as a therapeutic target for neurodegenerative diseases. Neurosci Lett. 2014;565:59-64.

18. Ishiki A, et al. Glial fibrillar acidic protein in the cerebrospinal fluid of Alzheimer's disease, dementia with Lewy bodies, and frontotemporal lobar degeneration. J Neurochem. 2016;136:258-61.

19. Abu-Rumeileh $S$, et al. CSF biomarkers of neuroinflammation in distinct forms and subtypes of neurodegenerative dementia. Alzheimer's Res Ther. 2019;12:2.

20. Elahi FM, et al. Plasma biomarkers of astrocytic and neuronal dysfunction in early- and late-onset Alzheimer's disease. Alzheimer's Dementia. 2019;16:681-95.

21. Oeckl P, et al. Glial Fibrillary Acidic Protein in Serum is Increased in Alzheimer's Disease and Correlates with Cognitive Impairment. J Alzheimer's Dis. 2019;67:481-8.

22. Asken BM, et al. Plasma Glial Fibrillary Acidic Protein Levels Differ Along the Spectra of Amyloid Burden and Clinical Disease Stage. J Alzheimer's Dis. 2020;78:265-76. 
23. Verberk IMW, et al. Combination of plasma amyloid beta(1-42/1-40) and glial fibrillary acidic protein strongly associates with cerebral amyloid pathology. Alzheimers Res Ther 118 (2020) doi:10.1186/s13195-020-00682-7.

24. Albert MS, et al. The diagnosis of mild cognitive impairment due to Alzheimer's disease: Recommendations from the National Institute on Aging-Alzheimer's Association workgroups on diagnostic guidelines for Alzheimer's disease. Alzheimer's Dementia. 2011;7:270-9.

25. Jessen $\mathrm{F}$, et al. A conceptual framework for research on subjective cognitive decline in preclinical Alzheimer's disease. Alzheimer's Dementia. 2014;10:844-52.

26. Molinuevo JL, et al. Implementation of subjective cognitive decline criteria in research studies. Alzheimer's Dementia. 2017;13:296-311.

27. Pusswald G, et al. Prevalence of mild cognitive impairment subtypes in patients attending a memory outpatient clinic-comparison of two modes of mild cognitive impairment classification. Results of the Vienna Conversion to Dementia Study. Alzheimer's Dementia. 2013;9:366-76.

28. Lehrner $\mathrm{J}$, et al. Awareness of memory deficits in subjective cognitive decline, mild cognitive impairment, Alzheimer's disease and Parkinson's disease. Int Psychogeriatr. 2015;27:357-66.

29. Kühner C, Bürger C, Keller F, Hautzinger M. Reliabilität und Validität des revidierten BeckDepressionsinventars (BDI-II). Nervenarzt. 2007;78:651-6.

30. Philippe $\mathrm{C}$, et al. Optimization of the radiosynthesis of the Alzheimer tracer 2-(4-N[11C]methylaminophenyl)-6-hydroxybenzothiazole ([11C]PIB). Appl Radiat Isotopes. 2011;69:12127.

31. Teunissen CE, Tumani H, Engelborghs S, Mollenhauer B. Biobanking of CSF: International standardization to optimize biomarker development. Clin Biochem. 2014;47:288-92.

32. Duits FH, et al. Diagnostic impact of CSF biomarkers for Alzheimer's disease in a tertiary memory clinic. Alzheimer's Dementia. 2015;11:523-32.

33. Vanmechelen E, et al. Quantification of tau phosphorylated at threonine 181 in human cerebrospinal fluid: a sandwich ELISA with a synthetic phosphopeptide for standardization. Neurosci Lett. 2000;285:49-52.

34. Vanderstichele $H$, et al. Standardization of measurement of $\beta$-amyloid(1-42) in cerebrospinal fluid and plasma. Amyloid. 2009;7:245-58.

35. Altmann P, et al. Serum neurofilament light chain withstands delayed freezing and repeated thawing. Sci Rep-uk. 2020;10:19982.

36. Youden WJ. Index for rating diagnostic tests. Cancer. 1950;3:32-5.

37. DeLong ER, DeLong DM, Clarke-Pearson DL. Comparing the Areas under Two or More Correlated Receiver Operating Characteristic Curves: A Nonparametric Approach. Biometrics. 1988;44:837.

38. Robin X, et al. pROC: an open-source package for R and $S+$ to analyze and compare ROC curves. Bmc Bioinformatics. 2011;12:77. 
39. Lindsay J, et al. Risk Factors for Alzheimer's Disease: A Prospective Analysis from the Canadian Study of Health and Aging. Am J Epidemiol. 2002;156:445-53.

40. Subramaniapillai S, Almey A, Rajah MN, Einstein G. Sex and gender differences in cognitive and brain reserve: Implications for Alzheimer's disease in women. Front Neuroendocrin. 2021;60:100879.

41. Frost GR, Li Y-M. The role of astrocytes in amyloid production and Alzheimer's disease. Open Biol. 2017;7:170228.

42. et al. Review: Astrocytes in Alzheimer\&apos;s disease and other age-associated dementias:a supporting player with a central role. Neuropath Appl Neuro 43, 281-298 (2017).

43. Verberk IMW, et al. Serum markers glial fibrillary acidic protein and neurofilament light for prognosis and monitoring in cognitively normal older people: a prospective memory clinic-based cohort study. Lancet Heal Longev. 2021;2:e87-95.

44. Chatterjee $P$, et al. Plasma glial fibrillary acidic protein is elevated in cognitively normal older adults at risk of Alzheimer's disease. Transl Psychiat. 2021;11:27.

45. Thebault S, Booth RA, Freedman MS. Blood Neurofilament Light Chain: The Neurologist's Troponin? Biomed. 2020;8:523.

46. Forgrave LM, Ma M, Best JR, DeMarco ML. The diagnostic performance of neurofilament light chain in CSF and blood for Alzheimer's disease, frontotemporal dementia, and amyotrophic lateral sclerosis: A systematic review and meta-analysis. Alzheimer's Dementia Diagnosis Assess Dis Monit. 2019;11:730-43.

47. Benedet $A L$, et al. Plasma neurofilament light associates with Alzheimer's disease metabolic decline in amyloid-positive individuals. Alzheimer's Dementia Diagnosis Assess Dis Monit. 2019;11:679-89.

48. Andersson E, et al. Blood and cerebrospinal fluid neurofilament light differentially detect neurodegeneration in early Alzheimer's disease. Neurobiol Aging. 2020;95:143-53.

49. Cicognola $\mathrm{C}$, et al. Plasma glial fibrillary acidic protein detects Alzheimer pathology and predicts future conversion to Alzheimer dementia in patients with mild cognitive impairment. Alzheimer's Res Ther. 2021;13:68.

50. Rojas JC, et al. Plasma neurofilament light chain predicts progression in progressive supranuclear palsy. Ann Clin Transl Neur. 2016;3:216-25.

51. Gisslén M, et al. Plasma Concentration of the Neurofilament Light Protein (NFL) is a Biomarker of CNS Injury in HIV Infection: A Cross-Sectional Study. Ebiomedicine. 2016;3:135-40.

52. Bergman J, et al Neurofilament light in CSF and serum is a sensitive marker for axonal white matter injury in MS. Neurology Neuroimmunol Neuroinflammation 3, NA; (2016).

53. Teitsdottir UD, et al. Association of glial and neuronal degeneration markers with Alzheimer's disease cerebrospinal fluid profile and cognitive functions. Alzheimer's Res Ther. 2020;12:92.

\section{Figures}




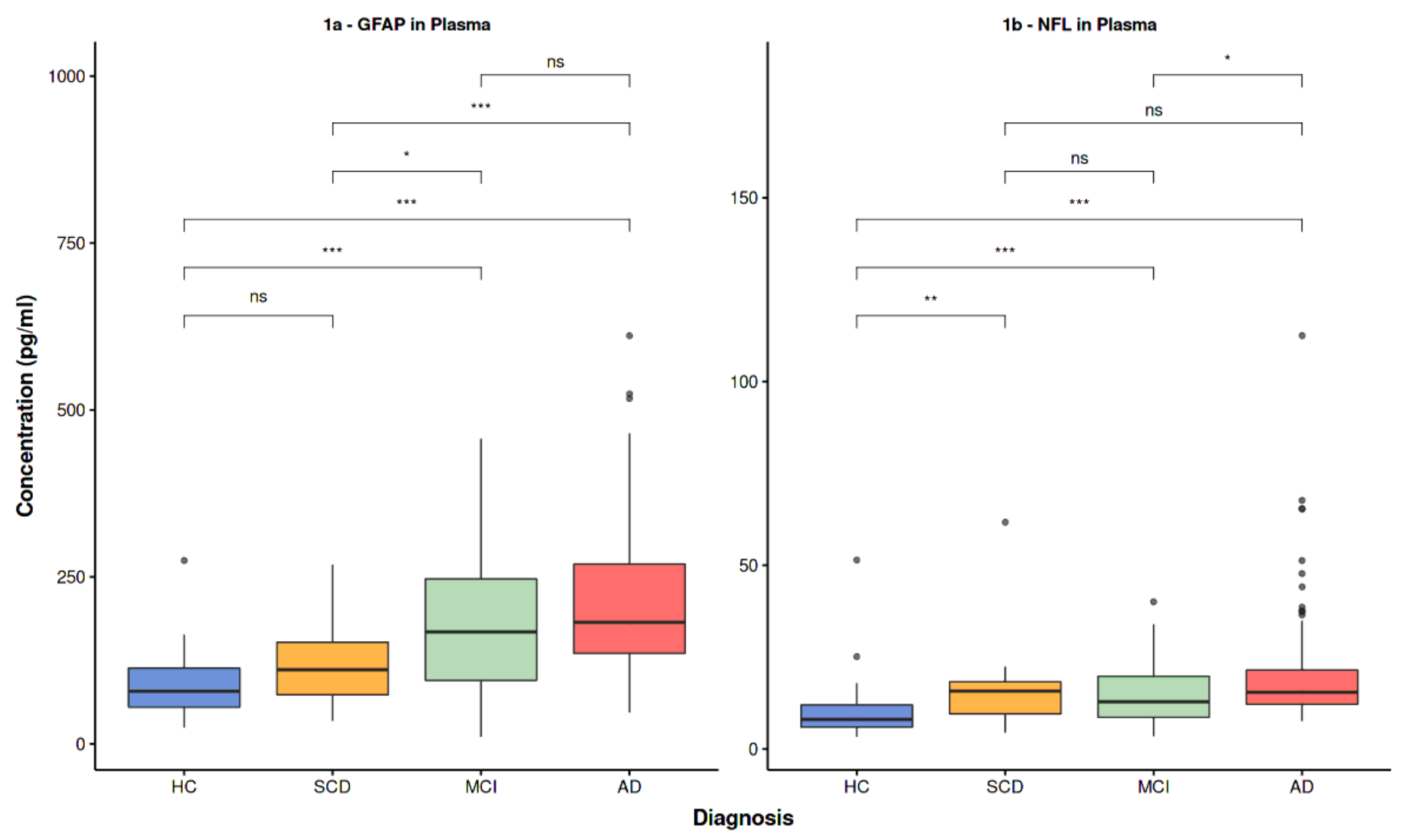

Figure 1

Concentration of GFAP (a) and NfL (b) in plasma among the four cohorts (HC, SCD, MCl, AD) Differences of biomarker concentration were calculated using Kruskal-Wallis Test, $p$ value is displayed as * $p<0.05$, $\star \star p<0.01, * \star \star p<0.001$, ns not significant HC healthy control, SCD subjective cognitive decline, $\mathrm{MCl}$ mild cognitive impairment, AD Alzheimer's disease, CSF cerebrospinal fluid, NfL neurofilament light chain, GFAP glial fibrillary acidic protein 

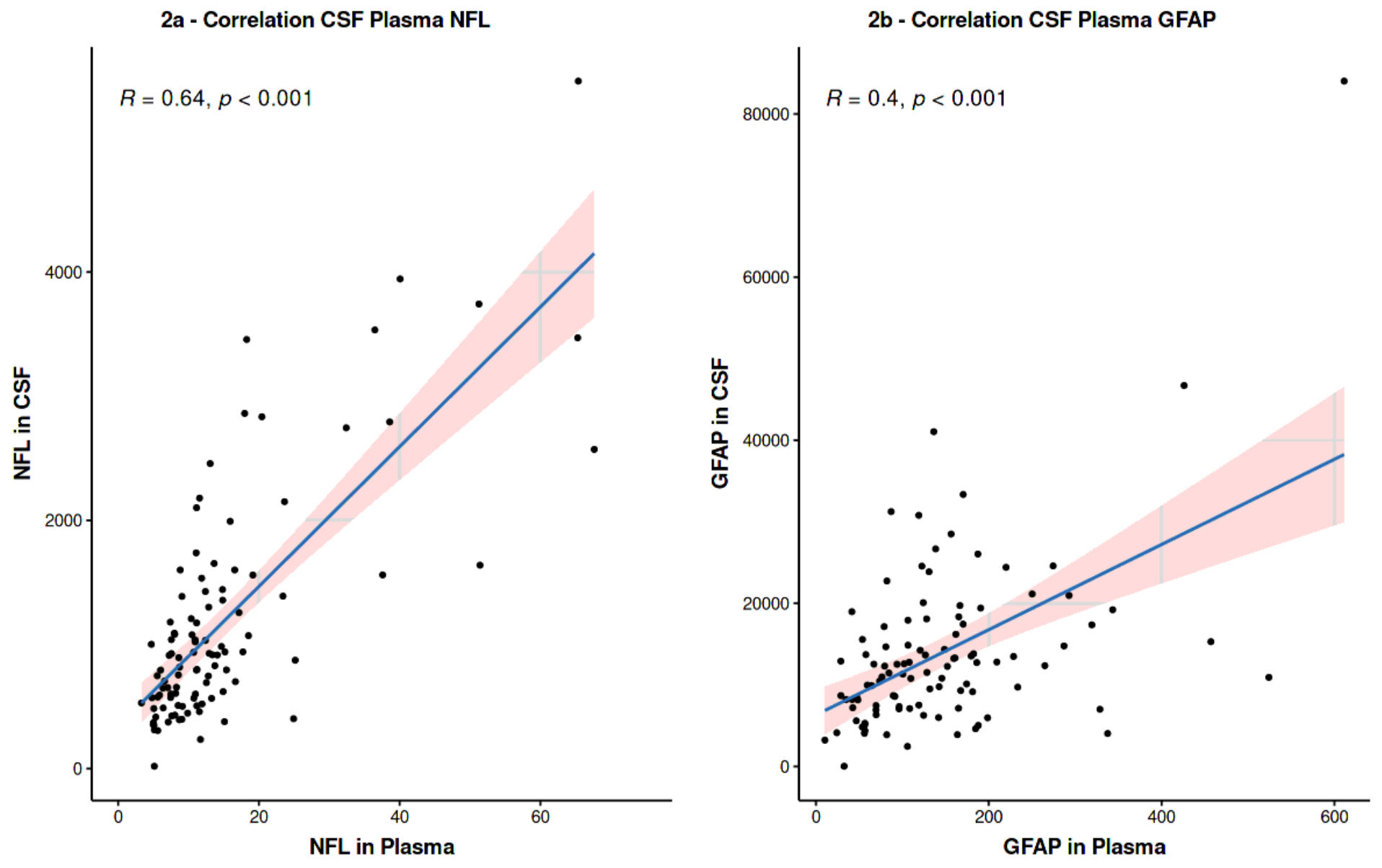

Figure 2

Correlation of NfL in CSF and plasma (a) and GFAP in CSF and plasma (b). Correlation was assessed using Spearman's rank correlation coefficient. CSF cerebrospinal fluid, $\mathrm{NfL}$ neurofilament light chain, GFAP glial fibrillary acidic protein 
a

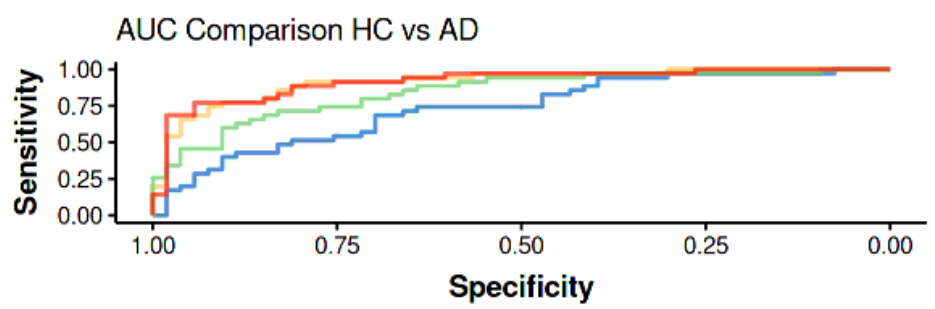

c

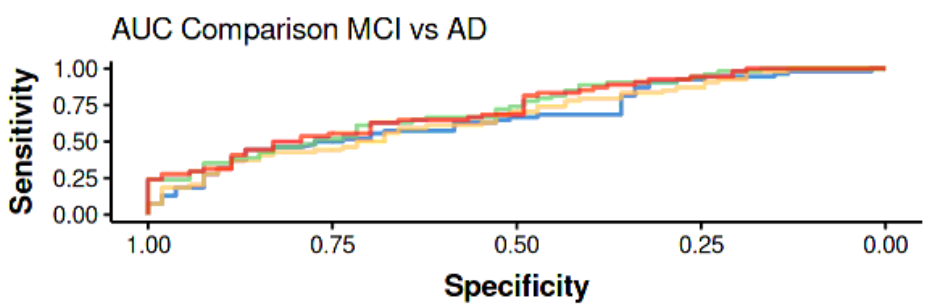

e

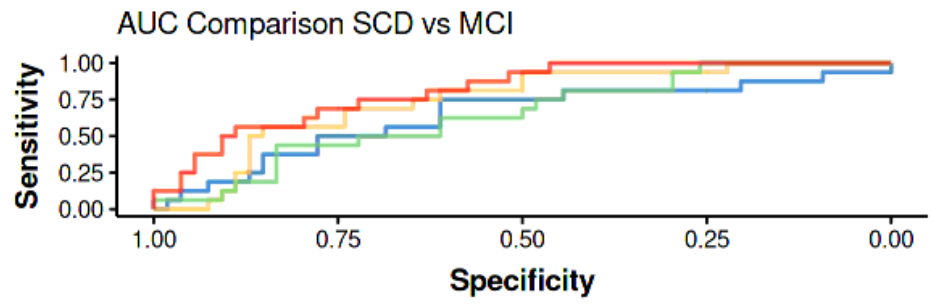

g

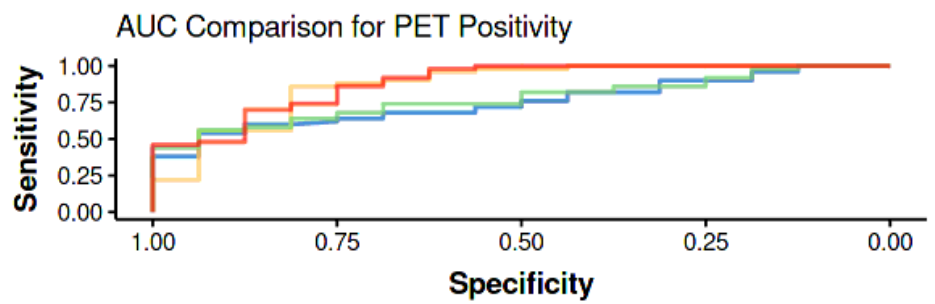

b

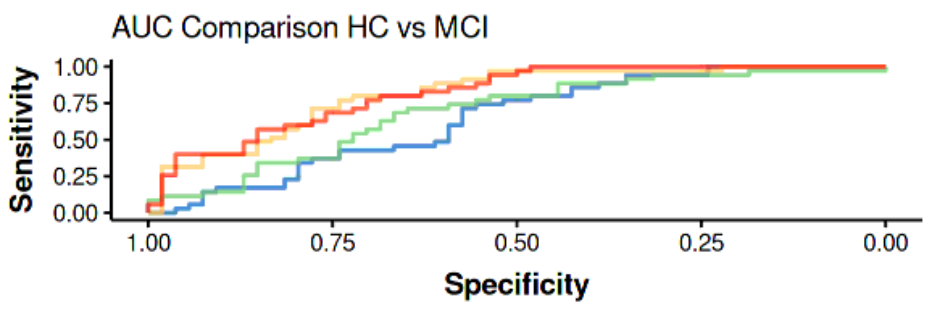

d
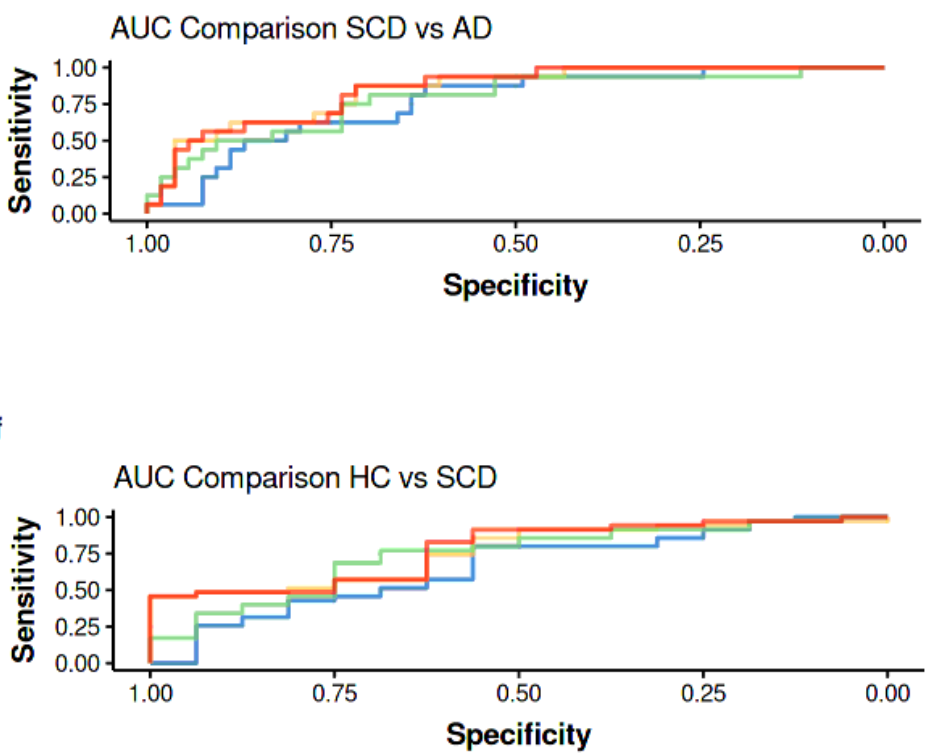

\section{Figure 3}

Receiver operating characteristic (ROC) curves for the diagnostic performance in distinguishing $\mathrm{HC}$ from $A D(a), H C$ from $M C l(b), M C l$ from $A D(c), S C D$ from $A D(d), S C D$ from $M C l(e), S C D$ from $H C$ (f), the prediction of amyloid positivity in our cohort $(\mathrm{g})$. The area under the curve (AUC) of each model was compared using DeLong's test for correlated AUC curves. The four panels analysed are called age+sex+APOE4 (blue), GFAP+ (i.e. age+sex+APOE4+GFAP, orange), NfL+ (i.e. age+sex+APOE4+NfL, green) and GFAP+NFL+ (age+sex+APOE4+GFAF+NfL, red) in this figure. $\mathrm{HC}$ healthy control, SCD 
subjective cognitive decline, $\mathrm{MCl}$ mild cognitive impairment, AD Alzheimer's disease, NfL neurofilament light chain, GFAP glial fibrillary acidic protein 